\title{
Observation of Postoperative Analgesia in Elderly Patients with PFNA By Routine Pulsed Lumbar Plexus Administration
}

\author{
Song Zhi-yong, Wang Qi \\ Department of Anesthesiology, Shouguang Hospital of Traditional Chinese Medicine, Shouguang, Shandong \\ Song Zhi-ying, E-mail: T5262337@126.com, https://orcid.org/0000-0003-1940-7217. \\ Qi Wang, https://orcid.org/0000-0002-9600-8490.
}

Received:1 December 2019 Accepted: 5 December 2019 Published: 31 December 2019

Cite this Article Song Zhi-yong, Wang Qi: Observation of postoperative analgesia in elderly patients with PFNA by routine pulsed lumbar plexus administration[J].Medical Research,2019.1(1):23-25, http://dx.doi.org/10.6913 /MRHK. 201912_1(1).0004

Copyright (C) 2020 Creative Publishing Co., Limited. All rights reserved. Email:kycbshk@gmail.com.

\begin{abstract}
Objective to observe the postoperative analgesia of elderly patients with PFNA by pulsed lumbar plexus administration. Methods: 90 patients with PFNA postoperative analgesia were selected and divided into three groups on average. The first group, 30 routine PCIA. The second group received PCEA analgesia after spinal anesthesia. In the third group, the intraoperative lumbar plexus nerve block was completed and the anesthetic catheter was indwelling. The drug administration mode was programmed pulse administration without background dose. The pain index, movement tissue index and the incidence of various adverse reactions were observed after PFNA.

Results the pain score of peripheral nerve block program pulse group was significantly lower than that of general anesthesia group, the incidence of motor tissue was lower than that of epidural group, and the incidence of side effects was lower than that of general anesthesia group and epidural group.

Conclusion patients with PFNA is mostly elderly patients with varying degrees of system disease and organ function degradation, extra week nerve block program pulse to VAS score is lower and less motor block, fewer side effects, is PFNA more effective postoperative analgesia mode, you can reply as soon as possible to the patient's activity ability, improve the prognosis of patients.
\end{abstract}

Key Words Observation of Postoperative Analgesia ; Elderly Patients;PFNA

China has fully entered the aging society. With the increase of age, the elderly are prone to femoral neck fracture, femoral trochanteric fracture, etc. These hip fractures are the most serious fracture types caused by fracture osteoporosis. After the fracture, the patient's mobility and quality of life are reduced. Moreover, elderly patients are prone to recurrent osteoporotic fractures after surgery, such as contralateral hip fractures. As the elderly patients have many complications before injury, it has a serious impact on the body. Elderly patients with progressive organ function decline and decompensation, are prone to complications of cardiopulmonary and renal diseases, and are prone to unpredicted adverse events related to drugs, anesthesia and surgery during perioperative period. For such patients, the short-term goal is to effectively relieve pain, restore the patients' living ability as soon as possible, and avoid bed rest and complications caused by it. Self-control analgesia method has the characteristics of reliable effect, quick effect, therefore in the process of the analgesic treatment of orthopaedic surgery, the way of the wider application range methods mainly include epidural drug dosing and intravenous drug delivery as well as the peripheral nerve block, dosing way 
Medical Research ISSN 2664-0333 Volume 1 No.1 PP.23-25 http://dx.doi.org/10.6913/MRHK.201912_1(1).0004

Song Zhi-yong, Wang Qi: Observation of postoperative analgesia in elderly patients with PFNA by routine pulsed lumbar plexus administration mostly load plus continuous as well as the additional mode of self-control analgesia, program pulse to drugs used in the study of postoperative analgesia. In this study, the analgesic effects and adverse reactions of PCEA and peripheral nerves in patients with postoperative analgesia of orthopedic PFNA under the mode of PCIA and programmed pulse administration were observed, providing a more suitable method for clinical management of postoperative pain of PFNA.

\section{GENERAL INFORMATION AND METHODS}

General data selection of 90 cases with PFNA in orthopaedic surgery, aged $60 \sim 90$ years old, ASA grade I III were randomly divided into PCIA group (group I), the PCEA group (group E) and peripheral nerve postoperative analgesia group ( $\mathrm{N}$ group), 30 people in each group. Exclusion criteria: if the original anesthesia method fails to change the anesthesia method, the postoperative analgesia score cannot be matched.

Anesthesia and analgesia methods epidural analgesia was performed in the PCEA group after the operation. The analgesia pump with a load of $1 \mathrm{mg}$ morphine was formulated with $0.125 \%$ bupivacaine $+0.5 \mathrm{ug} / \mathrm{ml}$ sufentanil. In the PCIA group, sufentanil 3-5ug analgesic formula was $2 \mathrm{ug} / \mathrm{ml}+$ tropisetron $10 \mathrm{mg}$ after general anesthesia. Each additional dose of the above two groups was $0.5 \mathrm{ml}$, and the locking time was 15 minutes. The analgesic and analgesic formula for peripheral nerve block was $0.15 \%$ bupivacaine with no load. The dosage was added once per hour for $4 \mathrm{ml}$ each time, and the dosage was not more than 30 seconds. The dosage was added for $3 \mathrm{ml}$ each time, and the locking time was $15 \mathrm{~min}$.

Visual analogue score (VAS) was observed and recorded at 2, 4, 8, 24 and 48 hours after the operation, indicating sharp pain when the result was 10 , satisfied with analgesia when the result was less than or equal to 3 , and painless during the operation and analgesia when the result was zero. Postoperative motor block was evaluated by modified Bromage score. No leg lift at level 1; Level 2 cannot extend the knees; Level 3 does not extend or bend the ankle. Meanwhile, the incidence of adverse reactions such as nausea and vomiting during analgesia was recorded. Postoperative recovery was followed up 1 week, 1 month and 6 months after surgery.

\section{STATISTICAL METHOD}

SPSS12.00 statistical software was used for data processing. The standard deviation of measurement indexes in this study was represented by $(x- \pm s)$, and variances were analyzed for various data in this study. For inter-group data, $\mathrm{t}$ was used to test, and $\mathrm{x}$ was used ${ }^{2}$ To test the counting data, and the comparison between them is statistically significant. $(\mathrm{P}<0.05)$.

\section{THE RESULTS}

In general, there were no statistically significant differences in age, weight and operation time between each group.

VAS scores of postoperative 4, 8 and 24 in group I were higher than those in group E and group $\mathrm{N}$, and there was no significant difference in scores at 2 and $48 \mathrm{~h}$ (table 1 ).

In the three groups, muscle strength of lower limbs could be completely recovered within $6 \mathrm{~h}$ after the operation. 3 patients in group E had motor block, 4 patients in group I had severe nausea, vomiting and itching, and no motor block and adverse reactions occurred in group $\mathrm{N}$ (table 1).

Table 1 postoperative pain scores and adverse reactions of patients in the three groups (case)

\begin{tabular}{c}
\hline The number of patients in the group was $2 \mathrm{~h}, 4 \mathrm{~h}, 8 \mathrm{~h}, 24 \mathrm{~h}$ and $48 \mathrm{~h}$ \\
\hline Group I $301 \pm 0.5^{\wedge} 24 \pm 0.5^{\wedge} 14 \pm 0.5^{\wedge} 11 \pm 0.5^{\wedge} 24^{\wedge} 3$ \\
Group E $301 \pm 0.5^{\wedge} 2 \pm 0.5^{\wedge} 24 \pm 0.5^{\wedge} 1 \pm 0.5^{\wedge} 11 \pm 0.5^{\wedge} 23^{\circ}$ \\
Group N $301 \pm 0.52 \pm 0.52 \pm 0.52 \pm 0.51 \pm 0.5$ \\
\hline
\end{tabular}




\section{DISCUSS}

PFNA is a common type of surgery in clinical practice, and most of the patients are elderly. Although this type of surgery has little trauma, it is characterized by severe pain in orthopedic surgery and requires early postoperative activities. However due to the old people in all kinds of physiological function gradually faded, and often accompanied by a variety of complications, it is to a certain extent of anesthesia and analgesia effect put forward higher request ${ }^{[1]}$, for such patients, short-term goal for effective analgesia, as soon as possible to restore the ability of the patient's life, avoid bed and the resulting complications; The long-term goal is to help patients return to their pre-injury mobility and function as much as possible and to avoid further falls and fractures ${ }^{[2,3]}$. According to relevant statistics, the mortality rate of elderly patients after injury is as high as $30 \%$. Therefore, people from all walks of life pay more and more attention to brittle fracture of the elderly.

Analgesia methods mainly include PCIA and PCEA. When patients use PCEA technology in the process of analgesia, they must learn how to properly use the analgesia pump. However, some patients may not want to control the analgesia by themselves due to fatigue and other reasons, and would rather leave these things to the doctor. In recent years, domestic and foreign studies on the use of PIEB + PCEA for analgesia have gradually increased. With the PIEB technique, the analgesia pump is programmed to be administered in a regular pulse mode rather than in a continuous infusion mode. The analgesia results are excellent, with few bursts of pain, few side effects, no serious complications and major technical errors, and high satisfaction.

PFNA patients are mostly elderly patients with different degrees of systemic diseases and organ function degradation, and peripheral nerve block procedure pulse drug VAS score is lower, less motor block, less side effects, which is a more effective analgesia mode after PFNA surgery, can restore the patient's activity ability as soon as possible, improve the prognosis of patients.

\section{REFERENCES}

1 Johansen A,Romundstad L,Nielsen CS,et al.Persistent postsurgical pain in ageneral population:prevalence and predictors in the Tromso study .Pain,2012,153(7):1390 1396

2 Barrington MJ, Olive D, Low K, et al.Continuous femoral nerve blockade or epidural analgesia after total knee replacement: a prospective randomized controlled trial .Anesth Analg,2005,101:1824-1829

3 Ashburn M,Caplan R,et al.Practice Guidelines for Acute Pain Management in Perioperative Setting. Anesthesuology, 2012,116(2): 248-273 